\title{
Pacemaker implantation performed by surgeons and cardiologists in a single tertiary centre: A quality assurance study
}

\author{
Sam Mirzaee ${ }^{1 *}$, Mohammad Alasti ${ }^{2}$ and Saeed Oraii ${ }^{3}$ \\ ${ }^{1}$ Azad University Tehran Medical Sciences, Tehran, Iran \\ ${ }^{2}$ Ahvaz Jundishapur University of Medical Sciences, Ahvaz, Iran \\ ${ }^{3}$ Tehran Arrhythmia Centre, Tehran, Iran
}

\begin{abstract}
Objectives: Quality assurance is an important component of health care system to ensure all patients receiving an equal and sufficient level of care. Pacemaker implantation is a routine procedure in cardiology practice, however the overall complications related to implantation has not been well defined.

Methods: We performed a retrospective quality assurance study on 413 subjects collecting data from all primary pacemaker implantations performed by cardiothoracic surgeons in theatre and cardiologist in EP laboratory over a transitional period in 1999-2001 in a large tertiary hospital.

Results: A total of 114 pacemakers had been implanted by surgeons ( $46.5 \%$ male, mean age $63.3 \pm 18.4)$ and 299 cases by cardiologists ( $63.9 \%$ male, mean age $63.9 \pm 18.2$ ). Indications for implantation were nearly similar with AV block as the most common cause in $77 \%$ and $75 \%$ during eras 1 and 2 respectively. Single chamber pacemakers included $86 \%$ of implants during era 1 compared to $54 \%$ during era $2(\mathrm{P}<0.01)$. Overall pacemaker malfunctions were $7.9 \%$ versus $1.7 \%$ during era 1 and era 2 respectively $(\mathrm{P}<0.01)$. Similarly, early re-do procedures were performed in $2.7 \%$ of implantations during era 1 compared to $0.7 \%$ during era 2 ( $\mathrm{P}<0.01)$.
\end{abstract}

Conclusions: Overall rate of complications in this study was comparable with data in current literatures, yet the rate of lead malfunction followed by redo procedure seemed to be lower by cardiologist. A sizable multicentre review considering evaluation of the individual's skills in pacemaker implantation is warranted.

\begin{abstract}
Abbreviations: CNS: Central Nervous System; DDD: DualChamber Pacemaker; EP: Electrophysiology; NMS: Neuro Mediated Syncope; NS: Not Significant; PM: Pacemaker; SSS: Sick Sinus Syndrome; VDD: Ventricular Pacing with Atrial tracking.
\end{abstract}

\section{Introduction}

The practice of permanent pacemaker implantation is more than five decades old but it has seen remarkable developments ever since its initiation [1]. Cardioverter defibrillator and cardiac resynchronization therapy are good examples of this advancement in the last two decades. Permanent pacemakers was historically implanted in operating rooms, special procedure laboratories or cardiac catheterization laboratories and different members of medical team including cardiothoracic surgeons, physicians or a trained clinician may have been involved [2]. However, the first line diagnosis, referral and continuity of care usually carry out by a trained cardiac team. Current evidence demonstrates shortage in non-technical skills have an important role in the rate of adverse events and complication in surgical procedures [3]. The rate of complications associated with pacemaker (PM) varies in the different literatures reported between a wide range of 4-12.6\% for early and up to $2.7 \%$ for late complications [4,5]. Complications related to pacemaker implantation has been studied in previous studies demonstrating higher mortality and cost burden on health system [6-8]. One may argue pacemaker implant should be performed by a medical practitioner who has been trained for both non-technical and technical aspects of pacing.
Previous studies have shown no differences in terms of efficacy or outcome whether PM was implanted in the operating room or in the cardiac electrophysiology (EP) laboratory [9-11]. However, the pacemaker implantation by EP specialist in cardiac laboratory carries a lower cost [12].

This study reviewing two eras of pacemaker implantation by cardiac surgeons in an operation room following a relatively abrupt change when a new pacemaker implantation service was stablished by a group of trained EP cardiologists in Rajaie Heart Centre, Tehran, Iran. In order to review the code of conduct, safety and patient's care, we audited two eras of pacing therapy in a transitional period when a group of EP trained cardiologists stablished an EP department and become in charge of pacing. At the first era pacemaker implantation was performed by cardiac surgeons in an operation room, whereas later on following a drastic change in PM services was run by a team of EP trained cardiologists.

\section{Methods}

We retrospectively collected, analyzed and compared the data from all patients who received PM implantation by cardiovascular surgeons

${ }^{\star}$ Correspondence to: Sam Mirzaee, Azad University Tehran Medical Sciences, Tehran, Iran, E-mail: sammirzaee@hotmail.com

Key words: pacemaker implantation, complications, quality assurance

Received: June 19, 2018; Accepted: June 26, 2018; Published: June 29, 2018 
during the first half of the year in 1999 with those of the first half of the year in 2001 where firstly PM implantation was commenced by cardiologists in EP laboratory. Data from the first era were extracted from medical records into a data collection form, whereas the data from the second era was collected from electronic medical records. Basic characteristics of all subjects, PM information, immediate and short term complications ( $<3$ months) were extracted for further analysis.

Any adverse events requiring further diagnostic test, reoperation or prolonged hospital stay was considered as complications. Bleeding was defined as any external bleeding which needed seeking medical attention. Development of sensing issues and high pacing thresholds requiring device reprogramming or the need for repositioning leads was demonstrated as lead failure/problem. Infection related to PM insertion defined as any of the following infective process: wound infection, device system infection, fever and positive blood cultures without foci elsewhere [13].

Statistics: Data analysis was carried out using the descriptive statistics, Chi-square and student t-test procedures available in SPSS 10.0. $P$ value less than 0.05 with $95 \%$ confidence interval considered as significant.

\section{Results}

\section{Patient characteristics}

In total $413 \mathrm{PM}$ implanted; 114 patients (46.5\% male, mean age $63.3 \pm 18.4)$ undergone implantation by surgeons and $299(63.9 \%$ male, mean age $63.9 \pm 18.2$ ) by cardiologists (Table 1 ). Syncope, presyncope and dizziness were the clinical presentation in $6 \%, 32 \%$ and $48 \%$ of patients during the surgical era and in $17 \%, 22 \%$ and $60 \%$ of patients respectively in the cardiologist era $(p=N S)$. Other less common clinical presentations were palpitation, fatigue equally distributed in both groups.

Underling heart diseases were reported as ischemic heart disease in $25 \%$ vs $18 \%$, hypertension in $32 \%$ vs $24 \%$, valvular heart disease in $5 \%$ vs $6 \%$ and congenital heart disease in $4 \%$ vs $4 \%$ of patients during the surgical and cardiologist eras respectively $(p=N S)$. AV block was the most common indication for implantation of pacemakers at both groups. It comprised the primary indication for $75 \%$ of patients in the surgical era and $77.1 \%$ in cardiologist era $(p=N S)$. Sick sinus syndrome was the next most common indication; $23 \%$ of patients in the surgical era and $20.1 \%$ in the cardiologist era $(p=N S)$. Cardiac abnormalities which lead to PM implantation are illustrated in Figure 1.

\section{Implantation procedure data}

Insertion site was the right side in $91.2 \%$ of patients during the surgical era while it was the left side in $96.6 \%$ during the cardiologist era. Both groups used the percutaneous transvenous method in all cases.

Table 1. Basic characteristics of participants

\begin{tabular}{|l|c|c|c|}
\hline Patients' Characteristics & Era 1 (n=299) & Era 2 (u=114) & P Value \\
\hline Age & $63.9 \pm 18.2$ & $63.3 \pm 18.4$ & NS qi \\
\hline Male & $63.90 \%$ & $46.50 \%$ & NS \\
\hline Single Chamber PM & $63.50 \%$ & $47.30 \%$ & 0.05 \\
\hline Dual Chamber PM & $47.70 \%$ & $13.80 \%$ & $<0.001$ \\
\hline Ischemic Heart Disease & $18.00 \%$ & $25 \%$ & NS \\
\hline Hypertension & $24.00 \%$ & $32 \%$ & NS \\
\hline High Degree AV block & $75.00 \%$ & $77.10 \%$ & NS \\
\hline
\end{tabular}

AV; Atrioventricular, PM; Pacemaker
Figure 1. Indication for implanting pacemaker

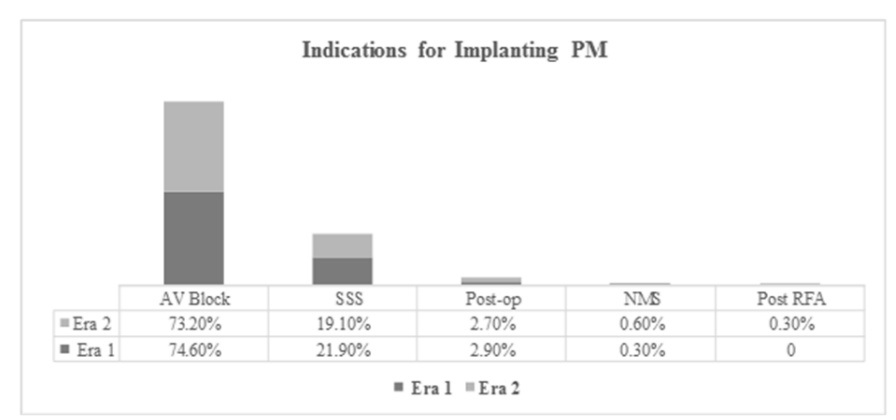

AV; Atrioventricular, NMS; Neuro Mediated Syncope, RFA; Radiofrequency ablation; SSS; Sick Sinus Syndrome

\section{Pacemaker modes}

$13.8 \%$ of pacemakers implanted during the surgical era were dual chamber pacemakers (including single pass VDD) compared to $47.7 \%$ during the cardiologist era $(p<0.001)$. Among single chamber devices, $47.3 \%$ of pacemakers implanted during the first era had rate responsive features compared to $63.5 \%$ during the second era $(p=0.05)$.

\section{Complications}

Post-procedure PM malfunction was documented in $7.9 \%$ of cases and overall complications during or after implantation were reported in $10.2 \%$ in surgical era.

During the cardiologist era pacemaker malfunction was reported in $1.7 \%(p<0.01)$ and overall complications was described in $2.1 \%$ of patients $(p=0.05)$ including hematoma $0.7 \%$, pneumothorax $0.3 \%$ and respiratory apnea $0.3 \%$. Reprogramming was necessary in $0.7 \%$ $(p<0.01)$, and chest tube drainage in $0.3 \%(\mathrm{P}=\mathrm{NS})$ (Figure 2). No procedure related death was reported during the two eras.

\section{Discussion}

This is a single center retrospective study from retrieved data with several caveats. The main finding of this retrospective study was the rate of complications in our centre is comparable with current literature (Figure 2) [4,5]. There were no significant differences in baseline characteristics of patients including age, sex distribution, underlying cardiac diseases and indications for PM implantation. However, it was a statistically significant differences in pacemaker types, insertion sites and rates of complications between two groups. In surgical era $86 \%$ of PM was VVI (single chamber) and 14\% dual chamber, whereas in cardiologist era $48 \%$ of PM was dual chamber (Table 1).

The rate of complications on lead malfunction seemed to be lower in cardiology group. This may reflect the role of an additional nontechnical EP based training and continuity of care by the operators. Consequently, this data suggests PM implantation by a trained multidisciplinary team may result in lower cost, hospital stay, overall patient's care and clinical outcome. To be able to generalise this result to all cardiac centres in our health system, a sizable multicentre quality assurance review including evaluation of individual skills in PM implantation is warranted.

\section{Limitations}

Several important limitations should be considered in the interpretation of our results. Firstly, it was a retrospective study with a relatively small sample size from retrieved data. Secondly the late 
Figure 2. Early complication post pacemaker implantation

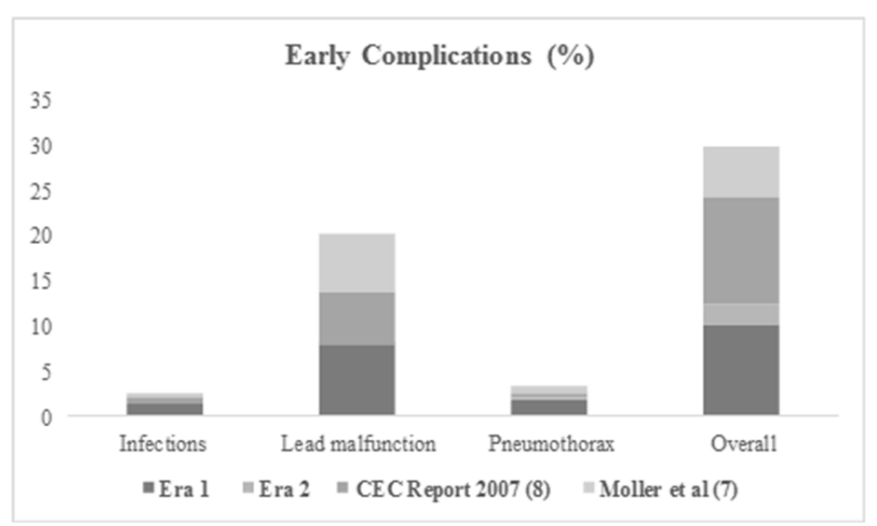

CEC; Clinical Excellence Commission

complications (> 3months) were poorly documented in both groups. Thirdly, there is a measurement biases in data collection, as cardiology data was collected from an electronic medical record versus data collection forms and medical charts in surgical group. Fourthly, the individual's skill and level of experience was not obtained in this study. Finally, no data collected on the socio-economic status of patient which in turn can affect the outcome, infection rate and further care as an important confounder.

\section{Conclusion}

These data advocate the importance of additional non-technical EP training in PM implanters which can result in better outcome. The authors suggest before endorsing a sensitive data such as PM procedure related complications, a large multicenter review is warrant.

\section{References}

1. Fu EY, Ellenbogen KA (2000) Management of atrial tachyarrhythmias in patients with implantable devices. Cardiology clinics 18: 37-53.

2. Asensio E, Mont L, Rubin JM, Herreros B, Ninot S, et al. (2000)[Prospective and comparative study of pacemaker implants carried out at the electrophysiology laboratory and the operating room]. Revista espanola de cardiologia 53: 805-859. [Crossref]

3. Agha RA, Fowler AJ, Sevdalis N (2015) The role of non-technical skills in surgery. Ann Med Surg (Lond) 4: 422-427. [Crossref]

4. Trohman RG, Kim MH, Pinski SL (2004) Cardiac pacing: the state of the art. Lancet 364: 1701-1719. [Crossref]

5. Pakarinen S, Oikarinen L, Toivonen L (2010) Short-term implantation-related complications of cardiac rhythm management device therapy: a retrospective singlecentre 1-year survey. Europace 12: 103-108. [Crossref]

6. Shakya S, Matsui H, Fushimi K, Yasunaga H (2017) In-hospital complications after implantation of cardiac implantable electronic devices: Analysis of a national inpatient database in Japan. J Cardiol 70: 405-410. [Crossref]

7. Møller M, Arnsbo P, Asklund M, Christensen PD, Gadsbøll N, et al. (2002) Quality assessment of pacemaker implantations in Denmark. Europace 4: 107-112. [Crossref]

8. Commission CE (2007) Review of Implantation Procedures for Permanent Pacemakers in NSW Public Hospitals, Public Report, Sydney.

9. García-Bolao I, Alegría E (1999) Implantation of 500 consecutive cardiac pacemakers in the electrophysiology laboratory. Acta Cardiol 54: 339-343. [Crossref]

10. Ferguson TB Jr, Lindsay BD, Boineau JP (1994) Should surgeons still be implanting pacemakers? Ann Thorac Surg 57: 588-596. [Crossref]

11. Miller GB, Leman RB, Kratz JM, Gillette PC (1988) Comparison of lead dislodgment and pocket infection rates after pacemaker implantation in the operating room versus the catheterization laboratory. Am Heart J 115: 1048-1051. [Crossref]

12. Yamamura KH, Kloosterman EM, Alba J, Garcia F, Williams PL, et al. (1999) Analysis of charges and complications of permanent pacemaker implantation in the cardiac catheterization laboratory versus the operating room. Pacing Clin Electrophysiol 22: 1820-1824. [Crossref]

13. Pakarinen S, Oikarinen L, Toivonen L (2010) Short-term implantation-related complications of cardiac rhythm management device therapy: a retrospective singlecentre 1-year survey. EP Europace 12: 103-108.

Copyright: (C2018 Mirzaee S. This is an open-access article distributed under the terms of the Creative Commons Attribution License, which permits unrestricted use, distribution, and reproduction in any medium, provided the original author and source are credited. 